\title{
Preditores cardiovasculares da mortalidade em idosos longevos
}

\author{
Cardiovascular mortality predictors \\ in the oldest old
}

Luiz Pedro Marafon 1

Ivana Beatrice Manica da Cruz 1,2

Carla Helena Augustin Schwanke 1,2

Emílio Hideyuki Moriguchi 1

\footnotetext{
1 Instituto de Geriatria e Gerontologia, Pontifícia Universidade Católica do Rio Grande do Sul. Av. Ipiranga 6690, Porto Alegre, RS 90610-000, Brasil. emoriguchi@pucrs.br 2 Faculdade de Biociências, Pontifícia Universidade Católica do Rio Grande do Sul. Av. Ipiranga 6681, Porto Alegre, RS 90619-900, Brasil.
}

\begin{abstract}
This article investigates the association between cardiovascular risk factors and cardiovascular morbidity and mortality in the oldest old. In 1996, 91\% of the population $\geq 80$ years of age from Veranópolis, Rio Grande do Sul State, Brazil, were evaluated to detect cardiovascular risk factors and morbidity. The sample was followed up for three years, with the assessment of deaths. The analysis was done using univariate statistics and multivariate logistic regression analysis. There were 41 deaths (21\%): 20 men and 21 women. Deaths were distributed by year as follow: 03 (7.3\%) in the first year, 08 (19.5\%) in the second, and 30 (73.2\%) in the third. There was a significant and independent association between death and the following variables: diastolic blood pressure (DBP), total cholesterol (TC), LDL-C, ApoA-I, prior stroke (CVA), right bundle branch block (RBBB), and left ventricular hypertrophy (LVH) by ECG. Survivors presented higher levels of DBP, TC, LDL-C, ApoA-I, CVA, RBBB and LVH. According to the multivariate analysis, the variables were independent risk factors for mortality. Cardiovascular risk factors appear to have a distinct impact on the oldest old.
\end{abstract}

Key words Aging Health; Risk Factors; Longevity

Resumo O objetivo deste trabalho é investigar a associação entre fatores de risco e morbidade cardiovascular com mortalidade em idosos longevos. Noventa e um por cento da população com idade $\geq 80$ anos $(n=193)$ de Veranópolis, Rio Grande do Sul, no ano de 1996, foram avaliados para a detecção de fatores de risco e morbidade cardiovascular. Acompanhou-se esta população durante 3 anos e registraram-se os casos de óbitos. Os dados foram analisados por análise univariada e multivariada por regressão logística. Ocorreram 41 (21\%) óbitos (20 homens e $21 \mathrm{mu}$ lheres). As mortes foram distribuídas ao longo do período estudado como se segue: 3 (7,3\%) no primeiro ano, 8 (19,5\%) no segundo ano e 30 (73,2\%) no terceiro ano. Observou-se associação significativa de óbito com as seguintes características: pressão arterial diastólica (PAD), colesterol total (CT), LDL-C, ApoA-I, acidente vascular encefálico prévio (AVC), bloqueio do ramo direito (BRD) e hipertrofia ventricular esquerda (HVE) ao ECG. Os sobreviventes apresentaram niveis mais elevados de PAD, CT, LDL-C e ApoA-I. AVC, BRD e HVE. A análise multivariada mostrou que as variáveis eram fatores de risco independentes. Os fatores de risco cardiovascular parecem atuar de forma diferenciada em longevos.

Palavras-chave Saúde do Idoso; Fatores de Risco Cardiovascular; Longevidade 


\section{Introdução}

O fenômeno do envelhecimento populacional, vivenciado por países desenvolvidos e em desenvolvimento, desperta a necessidade de pesquisas sobre idosos longevos ( $\geq 80$ anos). Isto porque este grupo etário apresenta características morfo-fisiológicas, psicológicas e socioeconômicas diferenciadas de outros indivíduos, inclusive idosos mais jovens (Hayflick, 1994).

No Brasil, investigações neste grupo etário são importantes, principalmente em estados com um contingente significativo de idosos. Este é o caso do Rio Grande do Sul. Durante a década de 90, existiam informações não oficiais de que Veranópolis teria uma das maiores expectativas de vida do Estado do Rio Grande do Sul. O cálculo da expectativa média de vida ao nascer do Rio Grande do Sul, realizado pelo Núcleo de Informações em Saúde - Rio Grande do Sul (1998) confirmou ser Veranópolis o município que, com mais de 17.000 habitantes, apresentava a maior expectativa média de vida ao nascer do Estado. Este índice, obtido a partir de dados de recontagem populacional de 1996 e da mortalidade do Departamento de Informática do SUS (DATASUS, 1996), estimou uma longevidade média de 77,7 anos em Veranópolis, ao passo que, no mesmo período, a expectativa média de vida no Estado foi de 71,8 anos e do Brasil de 67,7 anos. Dentro deste contexto, no ano de 1994 foi realizado um estudo piloto em Veranópolis, que lançou as bases para a implementação de uma investigação de base populacional que tinha como objetivo principal investigar variáveis associadas ao envelhecimento bem sucedido, mais especificamente estudando indivíduos com idade igual ou superior a 80 anos. Este projeto foi efetivamente implementado no ano de 1996.

Uma vez que, associado ao fenômeno do envelhecimento populacional, ocorre aumento na prevalência de doenças crônico-degenerativas associadas à idade, principalmente as doenças cardiovasculares (DCV) (Kashyap, 1989), a primeira etapa do Projeto Veranópolis foi dedicada a estudar variáveis associadas a fatores de risco ou fatores de proteção destas doenças em idosos longevos.

Neste artigo, descrevem-se a prevalência de fatores de risco clássicos de doenças cardiovasculares, bem como os fatores de risco emergentes e sua associação com a mortalidade de idosos longevos que residem no Município de Veranópolis, após três anos de seguimento (1996 a 1999).

\section{Métodos}

Delineamento e população investigada

O Município de Veranópolis foi escolhido por apresentar uma expectativa de vida similar à verificada em países desenvolvidos, uma homogeneidade étnica ( $95 \%$ da população descendem de imigrantes italianos) e um perfil sócio-econômico-cultural com poucas disparidades, principalmente em relação a idosos longevos ( $\geq 80$ anos). Como Veranópolis manteve um tamanho estável nos últimos 30 anos, possivelmente os efeitos de migração não foram predominantes no perfil demográfico. Na Tabela 1, sintetizam-se as características gerais do município, incluindo atividade econômica predominante, demografia e principais causas de óbito.

O delineamento foi do tipo prospectivo, observacional e descritivo-analítico em idosos longevos ( $\geq 80$ anos de idade). A identificação dos participantes foi feita a partir da recontagem populacional realizada pela Fundação Instituto Brasileiro de Geografia e Estatística (IBGE) em 1996, obtida na Secretaria do IBGE no Município. Foram identificados 213 moradores incluídos neste grupo etário, sendo que 193 participaram do estudo (91\%).

\section{Metodologia}

A primeira etapa do projeto consistiu em uma avaliação clínica, antropométrica e bioquímica de variáveis de risco cardiovascular (clássicas e emergentes) e presença de morbidades cardiovasculares prévias. A segunda etapa consistiu no levantamento de óbitos ao longo de três anos de seguimento da amostra investigada. O levantamento de óbitos foi feito junto à Secretaria de Saúde do município, que cadastra anualmente a mortalidade e os indicadores de saúde.

Detalhes da coleta dos dados clínicos e laboratoriais são descritos em Schwanke et al. (2002), incluindo anamnese, eletrocardiograma (ECG), análises bioquímicas e medida de pressão arterial. Além dos exames físicos, realizou-se uma entrevista estruturada, aplicada aos voluntários na presença do cuidador (respondente próximo). Caso o idoso não se recordasse e/ou tivesse dificuldade para relatar as questões solicitadas, a pergunta era novamente direcionada ao cuidador. Embora a coleta de dados tenha sido realizada durante três semanas no mês de julho de 1996, incluíram-se no estudo todos os idosos que tinham 80 anos completos até abril do mesmo ano. 
Detalhes sobre a coleta das variáveis investigadas também estão descritos em Schwanke et al. (2002). As principais variáveis investigadas foram: (1) antropométricas: foram avaliados o peso, altura, índice de massa corporal (IMC), calculado através da fórmula da razão do peso $(\mathrm{kg})$ pelo quadrado da altura $\left(\mathrm{m}^{2}\right)$ e a relação cintura/quadril (C/Q) conforme método descrito em Larsson et al. (1984); (2) pressão arterial sistêmica: obtida através da avaliação da pressão arterial sistólica (PAS) e diastólica (PAD) medida em um esfignomanômetro de mercúrio com manguito adequado para a circunferência do braço direito. A pressão foi avaliada três vezes em cada participante. Devido à possibilidade da existência de pseudo-hipertensão em idosos longevos, utilizou-se a manobra de Osler para a avaliação da pressão sistêmica; (3) variáveis bioquímicas: níveis de colesterol total, LDL-colesterol, HDL-colesterol, triglicerídeos e glicose (medidos em $\mathrm{mg} / \mathrm{dL}$ através de técnicas enzimáticas colorimétricas descritas em detalhe em Schwanke et al., 2002) e de ApoA-I e ApoB-100 (dosagens foram determinadas através de imunoturbidimetria, utilizando-se kits reagentes Sera-Pak fabricados pela Bayer Diagnostics Manufacturing-Bélgica); (4) variáveis do estilo de vida: a atividade física, o consumo alcóolico e o tabagismo foram investigados. Realizaram-se, na análise de variáveis bioquímicas, os seguintes pontos de corte: colesterol total ( $\geq 200 \mathrm{mg} / \mathrm{dL}$ ), ApoA-I ( $\geq 100 \mathrm{mg} / \mathrm{dL}$ ) (Contois et al., 1996a), ApoB-100 ( $\geq 120 \mathrm{mg} / \mathrm{dL}$ ) (Contois et al., 1996b). O grau de atividade física foi inferido a partir do cálculo do gasto energético em quilocalorias por semana (Kcal/sem) pelo método descrito em Dipietro et al. (1993); o consumo semanal de bebida alcóolica foi obtido através de relato verbal de quantos copos de bebida alcóolica eram ingeridos por semana. Após, calculou-se o total de gramas de álcool etílico ingerido utilizando-se como padrão doses de vinho, cerveja e destilado com aproximadamente $15 \mathrm{~g}$ de álcool absoluto conforme orientação do Serviço de Atenção ao Alcoolismo e Drogadição do Ministério da Saúde (Soibelman Jr., 1990).

Investigaram-se também os seguintes fatores de risco e morbidades cardiovasculares prévias: hipertensão arterial sistêmica (HAS), diabetes mellitus, obesidade, dislipidemia, claudicação intermitente, acidente vascular encefálico (AVC) prévio, cardiopatia definida pelo protocolo de Rose positivo e/ou alteração eletrocardiográfica: bloqueio de ramo direito (BRD), bloqueio de ramo esquerdo (BRE), hipertrofia ventricular esquerda (HVE) (Rose et al., 1982).

\begin{tabular}{|c|c|}
\hline \multicolumn{2}{|c|}{ Características gerais do Município de Veranópolis, Rio Grande do Sul, Brasil. } \\
\hline \multicolumn{2}{|l|}{ Características } \\
\hline Ano de fundação & 1884 \\
\hline Economia & Indústria \\
\hline Produto Interno Bruto (US\$) & 148.987 .147 \\
\hline Região geográfica & Serra \\
\hline Área $\left(\mathrm{km}^{2}\right)$ & 306,3 \\
\hline População & 18.122 \\
\hline Número de idosos longevos ( $\geq 80$ anos) & 213 \\
\hline Expectativa de vida ao nascer (anos) & 77,7 \\
\hline Masculina & 72,1 \\
\hline Feminina & 83,8 \\
\hline Coeficiente de mortalidade infantil (100 mil habitantes) & 0,001 \\
\hline Coeficiente de mortalidade geral (100 mil habitantes) & 5,59 \\
\hline Mortalidade total em 1996 (n) & 107 \\
\hline Principais causas de mortalidade & $\mathrm{n}(\%)$ \\
\hline Aparelho circulatório & $31(29)$ \\
\hline Neoplasias & $25(23)$ \\
\hline Aparelho respiratório & $8(7)$ \\
\hline Causas externas & $16(15)$ \\
\hline Outras causas & $27(26)$ \\
\hline
\end{tabular}

Fonte: Núcleo de Informação de Saúde - Rio Grande do Sul (1997); Instituto Brasileiro de Geografia e Estatística. Dados referentes aos anos de 1996 e1997.

Detalhes da coleta de dados e diagnóstico de HAS, diabetes mellitus, obesidade e dislipidemias estão descritos em Schwanke et al. (2002). Consideraram-se hipertensos indivíduos com PAS $\geq 140 \mathrm{mmHg}$ e PAD $\geq 90 \mathrm{mmHg}$ segundo o III Consenso Brasileiro de Hipertensão Arterial (Sociedade Brasileira de Hipertensão/Sociedade Brasileira de Cardiologia/Sociedade Brasileira de Nefrologia, 1998). O diagnóstico de AVC foi feito pelo exame clínico neurológico dos idosos por dois neurologistas. Doenças cardiovasculares também foram diagnosticadas por exames clínicos e eletrocardiográficos. Os achados eletrocardiográficos foram realizados por dois cardiologistas e analisados de forma independente e sem conhecimento prévio da história clínica dos idosos investigados. A presença de claudicação intermitente foi determinada através do módulo para avaliação de claudicação intermitente do Protocolo de Rose et al. (1982). Consideraram-se portadores de cardiopatias os idosos com Protocolo de Rose et al. (1982) positivo para angina e/ou para a possibilidade de infarto miocárdico e/ou com alteração eletrocardiográfica (Grupo ECG/Total). Para tanto, o Código de Minnesota (Rose et al., 1982) foi utilizado para classificar os achados eletrocardiográficos. Na ausência de Wolf- 
Parkinson-White (WPW) e de bloqueio de ramo esquerdo (BRE), considerou-se patológica a identificação das seguintes alterações da onda Q: parede ântero-lateral (DI, aVL, V6); parede inferior (DII, DIII, AVF); parede anterior (V1-V5). Na ausência de bloqueio de ramo direito (BRD) ou de bloqueio intraventricular, QRS com duração igual ou superior a 0,12 segundos, na maioria dos batimentos, em qualquer das derivações DI, DII, DIII, aVL ou aVF, prosseguiu-se a análise através da avaliação do ponto J, do seguimento ST e da onda T. Foram consideradas patológicas as alterações da onda T códigos 5-1 e 5-2, acompanhadas ou não de alterações do seguimento ST códigos 4-1-1 ou 4-1-2. As alterações eletrocardiográficas foram utilizadas para análise seguindo a estratificação descrita na Tabela 2.

\section{Análise estatística}

Na análise univariada, incluíram-se variáveis quantitativas comparadas entre o grupo de indivíduos que evoluíram para óbito e os que sobreviveram através do teste não-paramétrico de Mann-Whitney. As variáveis categóricas foram comparadas através do teste do qui-quadrado, tendo sido também calculados a razão de chance (RC) e o intervalo de confiança para cada variável. Consideraram-se significativas comparações com $p<0,05$. Posteriormente, realizou-se uma análise multivariada por regressão logística (método Foward Wald) onde todas variáveis que, na análise univariada, apresentaram valores de $\mathrm{p} \leq 0,20$ foram incluídas no modelo. Todos os modelos de equação testados foram corrigidos para sexo e idade. A

Tabela 2

Alterações eletrocardiográficas utilizadas para o diagnóstico de cardiopatias em idosos longevos de Veranópolis, Rio Grande do Sul, Brasil.

\begin{tabular}{ll}
\hline Estratificação por grupo & Diagnóstico \\
\hline ECG/QQS & Portadores de onda $\mathrm{Q}$ e/ou de complexos $\mathrm{SS}$ \\
ECG/Tiso & Portadores de alterações em onda T isolada \\
ECG/QQST & $\begin{array}{l}\text { Portadores de ondas } \mathrm{Q} \text { e/ou de complexos } \mathrm{QS}, \\
\text { mais alterações na onda } \mathrm{T}\end{array}$ \\
ECG/BRE & Portadores de bloqueio de ramo esquerdo \\
ECG/BRD & Portadores de bloqueio de ramo direito \\
ECG/HVE & Portadores de HVE \\
ECG/Total & Portadores de quaisquer das alterações acima \\
\hline
\end{tabular}

$E C G$ = Eletrocardiograma; $Q Q S$ = Ondas eletrocardiográficas do seguimento; QQST = Ondas eletrocardiograficas do seguimento T; BRE = bloqueio de ramo esquerdo; $\mathrm{BRD}=$ bloqueio de ramo direito; $\mathrm{HVE}=$ hipertrofia ventricular esquerda. idade foi utilizada como variável contínua no modelo.

\section{Aspectos éticos}

Para sua realização, o presente estudo foi aprovado pelo Comitê de Ética em Pesquisa do Hospital São Lucas da Pontifícia Universidade Católica do Rio Grande do Sul, tendo cumprido todos princípios éticos contidos na Declaração de Helsinki.

\section{Resultados}

Dos 193 idosos avaliados, 69 eram do sexo masculino $(35,8 \%)$ e 124 do sexo feminino $(64,2 \%)$. Após três anos de seguimento, ocorreram 41 óbitos, representando $21 \%$ da amostra inicial, sendo 20 homens e 21 mulheres, não havendo diferença estatisticamente significativa entre os sexos $(p=0,07)$. Na Tabela 3, estão resumidos os principais resultados.

A distribuição de mortalidade ao longo do período de seguimento ocorreu do seguinte modo: 03 (7,3\%) óbitos no primeiro ano, 08 (19,5\%) e $30(73,2 \%)$ no terceiro ano.

Os indivíduos que foram a óbito apresentaram significativamente menores níveis de PAD, CT, LDL-C, ApoA-I e atividade física. A comparação estatística do HDL-C ficou no limite da significância. O nível de HDL-C tendeu a ser menor nos idosos que foram a óbito.

Compararam-se, na amostra investigada, fatores de risco e morbidades cardiovasculares. Os resultados, apresentando freqüência e razão de chance, constam da Tabela 4 .

Observaram-se freqüências significativamente mais altas em idosos longevos que foram a óbito para as seguintes variáveis: BRD, HVE, AVC, indivíduos com níveis de ApoA-I inferiores a $100 \mathrm{mg} / \mathrm{dL}$ e de CT inferiores a $200 \mathrm{mg} / \mathrm{dL}$.

Uma análise multivariada foi realizada para testar a presença de fatores intervenientes nos resultados obtidos através da análise univariada (Tabela 5).

As variáveis BRD, HVE, AVC, PAD, ApoA-I e $\mathrm{CT}>200$ foram consideradas fatores independentes da idade e de outras variáveis incluídas na equação associados à mortalidade.

\section{Discussão}

A partir da revisão da literatura, o estudo realizado pode ser considerado como a primeira investigação de fatores de risco e morbidades cardiovasculares após três anos de seguimento 
Comparação entre variáveis biológicas, bioquímicas e do estilo de vida entre idosos longevos vivos e mortos, após três anos de seguimento.

\begin{tabular}{|c|c|c|c|c|}
\hline Variáveis & Desfecho & $\mathbf{n}$ & Média $\pm d p$ & $p$ \\
\hline \multirow[t]{2}{*}{ Idade (anos) } & Vivos & 152 & $83,53 \pm 3,31 a$ & 0,555 \\
\hline & Mortos & 41 & $83,88 \pm 3,42$ & \\
\hline \multirow[t]{2}{*}{$\operatorname{IMC}\left(\mathrm{Kg} / \mathrm{m}^{2}\right)$} & Vivos & 152 & $26,62 \pm 4,91$ & 0,498 \\
\hline & Mortos & 41 & $26,90 \pm 3,69$ & \\
\hline \multirow[t]{2}{*}{$\mathrm{C} / \mathrm{Q}(\mathrm{cm})$} & Vivos & 152 & $0,90 \pm 0,07$ & 0,762 \\
\hline & Mortos & 40 & $0,90 \pm 0,07$ & \\
\hline \multirow[t]{2}{*}{ PAS (mmHg) } & Vivos & 152 & $169,18 \pm 24,54$ & 0,613 \\
\hline & Mortos & 41 & $167,01 \pm 27,76$ & \\
\hline \multirow[t]{2}{*}{ PAD (mmHg) } & Vivos & 152 & $91,97 \pm 12,78$ & 0,01 \\
\hline & Mortos & 41 & $87,42 \pm 14,33$ & \\
\hline \multirow[t]{2}{*}{$\mathrm{CT}(\mathrm{mg} / \mathrm{dL})$} & Vivos & 152 & $217,07 \pm 47,20$ & 0,001 \\
\hline & Mortos & 41 & $189,43 \pm 45,65$ & \\
\hline \multirow[t]{2}{*}{$\mathrm{HDL}-\mathrm{C}(\mathrm{mg} / \mathrm{dL})$} & Vivos & 149 & $47,10 \pm 13,15$ & 0,090 \\
\hline & Mortos & 40 & $41,16 \pm 10,42$ & \\
\hline \multirow[t]{2}{*}{ LDL-C (mg/dL) } & Vivos & 149 & $143,16 \pm 42,10$ & 0,001 \\
\hline & Mortos & 40 & $121,66 \pm 43,44$ & \\
\hline \multirow[t]{2}{*}{ TG (mg/dL) } & Vivos & 151 & $135,63 \pm 60,83$ & 0,796 \\
\hline & Mortos & 41 & $137,53 \pm 81,55$ & \\
\hline \multirow[t]{2}{*}{ Glicemia (mg/dL) } & Vivos & 152 & $94,83 \pm 25,64$ & 0,412 \\
\hline & Mortos & 41 & $101,27 \pm 31,36$ & \\
\hline \multirow[t]{2}{*}{ ApoA-I (mg/dL) } & Vivos & 152 & $169,95 \pm 34,07$ & 0,001 \\
\hline & Mortos & 41 & $150,02 \pm 28,75$ & \\
\hline \multirow[t]{2}{*}{ ApoB-100 (mg/dL) } & Vivos & 151 & $89,07 \pm 21,36$ & 0,092 \\
\hline & Mortos & 41 & $82,51 \pm 19,82$ & \\
\hline \multirow[t]{2}{*}{ Atividade física (Kcal/sem) } & Vivos & 147 & $5799,98 \pm 4919,73$ & 0,042 \\
\hline & Mortos & 34 & $3918,13 \pm 3043,07$ & \\
\hline \multirow[t]{2}{*}{ Consumo alcoólico (g/sem) } & Vivos & 18 & $202,72 \pm 186,55$ & 0,713 \\
\hline & Mortos & 78 & $208,84 \pm 169,99$ & \\
\hline
\end{tabular}

a Variáveis quantitativas comparadas entre vivos e mortos pelo teste não paramétrico Mann-Whitney; significância: $p<0,05$.

IMC = índice de massa corporal; C/Q = relação cintura quadril; PAS = pressão arterial sistólica;

$\mathrm{PAD}=$ pressão arterial diastólica; $\mathrm{CT}=$ colesterol total; $\mathrm{TG}=$ triglicerídio.

em uma coorte de idosos longevos no Brasil. Após três anos de seguimento de idosos longevos residentes no Município de Veranópolis, onde foi investigada a influência de fatores de risco e morbidades cardiovasculares com a mortalidade, seis variáveis apresentaram associação significativa e independente. Duas variáveis relacionadas com alterações eletrocardiográficas (BRD e HVE), duas variáveis associadas com o perfil lipídico e a pressão arterial sistêmica (CT, ApoA-I, PAD) e uma à morbidade (AVC).

Dos resultados obtidos destaca-se a falta de associação da mortalidade com fatores de risco clássicos para doenças cardiovasculares, como obesidade, hipertensão sistêmica, diabetes, dislipidemia, tabagismo e sedentarismo. Esta não associação tem sido previamente re- latada na literatura. Alencar et al. (2000), em um estudo sobre fatores de risco para aterosclerose e associação com suas complicações, em idosos com média de idade de 76 anos, não encontraram diferenças entre obesos e não obesos. Este foi um estudo transversal e, portanto, não avaliou a mortalidade. Gruen \& Connor (1996), em The Rancho Bernardo Study, avaliaram idosos com média de idade de 80 anos e tampouco encontraram associação entre obesidade (avaliada por IMC e relação C/Q) e mortalidade. Os autores questionaram se a obesidade representaria um fator de risco cardiovascular importante também para os idosos acima de 80 anos. Considerando os resultados aqui obtidos, similares aos descritos no estudo citado, pareceria que a obesidade tende a per- 
Comparação dos fatores de risco (clássicos e emergentes) e morbidades cardiovasculares em idosos longevos vivos e mortos, após três anos de seguimento.

\begin{tabular}{|c|c|c|c|c|c|}
\hline Variáveis & Desfecho & n (\%) & RC & IC $95 \%$ & $p$ \\
\hline \multicolumn{6}{|l|}{$\begin{array}{l}\text { Alterações } \\
\text { eletrocardiográficas }\end{array}$} \\
\hline \multirow[t]{2}{*}{ ECG/QQS } & Vivos & $7(4,6)$ & 3,117 & $0,921-10,435$ & 0,121 \\
\hline & Mortos & $5(13,2)$ & & & \\
\hline \multirow[t]{2}{*}{ ECG/Tiso } & Vivos & $5(3,3)$ & 1,034 & $1,004-1,0065$ & 0,582 \\
\hline & Mortos & $0(0)$ & & & \\
\hline \multirow[t]{2}{*}{ ECG/OOST } & Vivos & $13(18,6)$ & 1,608 & $0,536-4,828$ & 0,361 \\
\hline & Mortos & $5(13,2)$ & & & \\
\hline \multirow[t]{2}{*}{$\mathrm{ECG} / \mathrm{BRE}$} & Vivos & $7(4,6)$ & 1,143 & $0,228-5,737$ & 0,873 \\
\hline & Mortos & $2(5,3)$ & & & \\
\hline \multirow[t]{2}{*}{ ECG/BRD } & Vivos & $3(2,0)$ & 1,475 & $1,701-32,844$ & 0,009 \\
\hline & Mortos & $5(13,2)$ & & & \\
\hline \multirow[t]{2}{*}{$\mathrm{ECG} / \mathrm{HVE}$} & Vivos & $6(4,0)$ & 3,662 & $1,054-12,724$ & 0,046 \\
\hline & Mortos & $5(13,2)$ & & & \\
\hline \multirow[t]{2}{*}{ ECG/Total } & Vivos & $29(19,2)$ & 2,454 & $1,132-5,1319$ & 0,036 \\
\hline & Mortos & $14(36,8)$ & & & \\
\hline \multicolumn{6}{|l|}{ Riscos e morbidades } \\
\hline \multirow[t]{2}{*}{ IAM } & Vivos & $5(3,3)$ & 1,508 & $0,282-8,069$ & 0,642 \\
\hline & Mortos & $2(4,9)$ & & & \\
\hline \multirow[t]{2}{*}{ Claudicação } & Vivos & $13(8,6)$ & 0,548 & $0,119-2,533$ & 0,742 \\
\hline & Mortos & $2(4,9)$ & & & \\
\hline \multirow[t]{2}{*}{ AVC } & Vivos & $4(2,6)$ & 5,139 & $1,313-20,108$ & 0,022 \\
\hline & Mortos & $5(12,2)$ & & & \\
\hline \multirow[t]{2}{*}{ Dislipidemia } & Vivos & $24(15,7)$ & 0,747 & $0,266-2,095$ & 0,756 \\
\hline & Mortos & $5(12,2)$ & & & \\
\hline \multirow[t]{2}{*}{ HAS } & Vivos & $75(49,3)$ & 0,729 & $0,361-1,472$ & 0,479 \\
\hline & Mortos & $17(41,5)$ & & & \\
\hline \multirow[t]{2}{*}{ Diabetes mellitus } & Vivos & $22(15,3)$ & 1,142 & $0,450-2,898$ & 0,972 \\
\hline & Mortos & $7(17,1)$ & & & \\
\hline \multirow[t]{2}{*}{ Obesidade } & Vivos & $66(43,4)$ & 1,509 & $0,755-3,016$ & 0,322 \\
\hline & Mortos & $22(53,7)$ & & & \\
\hline \multirow[t]{2}{*}{ Tabagismo } & Vivos & $9(6,3)$ & 1,167 & $0,301-4,525$ & 0,826 \\
\hline & Mortos & $3(17,3)$ & & & \\
\hline \multirow{2}{*}{$\begin{array}{l}\text { Atividade física esperada } \\
(\geq 3.000 \mathrm{Kcal} / \mathrm{semana})\end{array}$} & Vivos & $43(18,9)$ & 1,974 & $1,000-4,244$ & 0,058 \\
\hline & Mortos & $15(44,1)$ & & & \\
\hline \multirow[t]{2}{*}{ ApoA-I ( $\geq 100 \mathrm{mg} / \mathrm{dL})$} & Vivos & $4(2,6)$ & 7,618 & $2,110-27,502$ & 0,002 \\
\hline & Mortos & $7(17,1)$ & & & \\
\hline \multirow[t]{2}{*}{ ApoB-100 ( $\geq 120 \mathrm{mg} / \mathrm{dL})$} & Vivos & $45(28,8)$ & 1,112 & $0,917-1,348$ & 0,426 \\
\hline & Mortos & $9(22,0)$ & & & \\
\hline \multirow[t]{2}{*}{$\mathrm{CT}(\geq 200 \mathrm{mg} / \mathrm{dL})$} & Vivos & $58(31,2)$ & 2,809 & $1,375-5,741$ & 0,004 \\
\hline & Mortos & $26(63,4)$ & & & \\
\hline
\end{tabular}

$\mathrm{N}=$ tamanho da amostra; $\mathrm{RC}=$ razão de chance obtida na análise univariada; nível de significância $=p<0,05$. IAM = infarto agudo do miocárdio; $E C G$ = Eletrocardiograma; $Q Q S$ = Ondas eletrocardiográficas do seguimento; QQST = Ondas eletrocardiográficas do seguimento T; BRE = bloqueio de ramo esquerdo;

$\mathrm{BRD}=$ bloqueio de ramo direito; HVE = hipertrofia ventricular esquerda; HAS = hipertensão arterial sistêmica; AVC = acidente vascular encefálico prévio. 
der o seu poder de associação em idosos longevos. Ao contrário, existem evidências que mostram que a perda de peso estaria relacionada com uma maior carga de morbi-mortalidade nesta faixa etária.

Observou-se, aqui, associação entre mortalidade e níveis baixos de PAD, porém, não se identificou associação da mortalidade com os níveis de PAS e HAS. Como previamente comentado, a falta de associação entre hipertensão e mortalidade em idosos longevos encontrada também já foi previamente relatada na literatura. Um estudo de seguimento durante 14 anos realizado na Itália com 5.185 indivíduos com idade entre 22 a 95 anos, hipertensos e normotensos, não conseguiu predizer mortalidade associada à hipertensão para os indivíduos com idade superior a 80 anos. No caso, os autores encontram associação entre hipertensão e mortalidade somente para mulheres com idade $<80$ anos (Casiglia et al., 2002).

Entretanto, é importante ter-se cautela com tais resultados. Isto porque, se considerarmos que o processo de envelhecimento está associado ao enrijecimento da parede arterial, essa alteração anatômica poderia ocasionar uma superestimativa dos níveis pressóricos à aferição indireta. Dessa forma, poderíamos estar classificando erroneamente indivíduos normais como hipertensos; justificando assim o bom prognóstico dos "níveis pressóricos mais elevados" na população de longevos sobreviventes (Mencken, 2000). Talvez este problema pudessem ser contornado se outros critérios para avaliação da pressão forem desenvolvidos para a avaliação da pressão arterial sistêmica de idosos longevos. Entretanto, como tais parâmetros ainda não foram totalmente desenvolvidos, na tentativa de minimizar o problema, realizou-se, aqui, a manobra de Osler em todos os participantes.

Paradoxalmente, os níveis médios de PAD, encontrados nos idosos longevos sobreviventes de Veranópolis apresentaram-se maiores do que os dos idosos que foram a óbito. Porém, tais resultados parecem estar em consonância com os descritos em outros estudos populacionais em idosos, sugerindo uma sobrevida maior para os longevos com níveis pressóricos um pouco mais elevados do que aqueles considerados ideais para as populações mais jovens (Kannel \& D’Agostino, 1997; Mattila et al., 1998; Tervahauta et al., 1993). Em relação a esta constatação, poderíamos formular o seguinte questionamento: existiria associação entre a presença de BRD e/ ou HVE ao ECG, a PAD e a mortalidade? No estudo, não foi observada tal associação na análise de regressão logística. Ao

\begin{tabular}{|c|c|c|}
\hline \multicolumn{3}{|c|}{$\begin{array}{l}\text { Análise de regressão logística para as variáveis } \\
\text { incluídas na equação. }\end{array}$} \\
\hline Variáveis & $p$ & $\mathrm{R}$ \\
\hline $\mathrm{CT}$ & 0,0011 & 0,2344 \\
\hline ApoA-I & 0,0001 & 0,2956 \\
\hline LDL-C & 0,0082 & 0,1771 \\
\hline ECG/BRD & 0,0006 & 0,2478 \\
\hline PAD & 0,0233 & 0,1408 \\
\hline AVC & 0,0329 & 0,1267 \\
\hline ECG/Total & 0,0098 & 0,1714 \\
\hline Atividade física & 0,1267 & 0,0457 \\
\hline ECG/HVE & 0,0133 & 0,1613 \\
\hline HDL-C & 0,0037 & 0,2011 \\
\hline ApoB-100 & 0,1645 & 0,0000 \\
\hline ECG/QQS & 0,1992 & 0,0000 \\
\hline Idade & 0,7630 & 0,0000 \\
\hline \multicolumn{3}{|c|}{$\begin{array}{l}\mathrm{p}=\text { nível de significância; } \mathrm{R}=\text { coeficiente de } \\
\text { correlação; } \chi^{2}=41,807 ; \text { graus de liberdade }=13 . \\
\mathrm{P}=0,0001 . \mathrm{A} \text { idade e a pressão arterial } \\
\text { diastólica foram incluídas na equação como } \\
\text { variáveis contínuas. } \\
\mathrm{CT}=\text { colesterol total; ECG = eletrocardiograma; } \\
\text { BRD = bloqueio do ramo direito; PAD = pressão } \\
\text { arterial diastólica; } \mathrm{AVC}=\text { acidente vascular encefálico } \\
\text { prévio; HVE = hipertrofia ventricular esquerda. }\end{array}$} \\
\hline
\end{tabular}

contrário, as três variáveis demonstraram estar associadas de forma independente uma das outras com a mortalidade. Entretanto, como o número de indivíduos investigados era pequeno, este fator limitante pode estar mascarando tal associação. O aumento do número de óbitos no seguimento do estudo provavelmente permitirá esclarecer se esta condição é realmente a mais provável.

Também foi observada associação entre níveis elevados de CT, LDL-C e de ApoA-I com menor risco de morte. No caso, o CT e o LDL-C apresentaram valores médios significativamente mais elevados no grupo de sobreviventes. Outros estudos populacionais observaram comportamento semelhante dos níveis de colesterol entre idosos longevos (Kronmal et al., 1993; Tervahauta et al., 1993; Weverling-Rijnsburger et al., 1997).

Em nosso estudo, ao realizarmos a análise multivariada, observamos que o CT e o LDL-C foram marcadores de mortalidade independente das demais variáveis analisadas. Em nossa amostra, aqueles idosos com o CT abaixo de $200 \mathrm{mg} / \mathrm{dL}$ apresentaram risco 2,8 vezes maior de morrer em três anos, quando comparados àqueles com o CT > 200mg/dL.

Algumas investigações têm relacionado o aumento da mortalidade com níveis baixos de 
CT em idosos como decorrência de patologias como câncer ou doenças infecciosas. A deficiência nutricional, provocada por tais doenças, poderia explicar a associação do CT baixo com a mortalidade aumentada. Em nosso estudo, não investigamos a presença de neoplasias ou de doenças infecciosas. No entanto, não havia indivíduos hospitalizados integrando a amostra, demonstrando existir aparentemente razoáveis condições de saúde entre os participantes. Porém, a não investigação da presença comprovada de neoplasias pode ser considerada como um fator limitante do estudo realizado.

Mesmo assim, como as neoplasias podem permanecer em uma fase subclínica relativamente longa, seria difícil uma cobertura diagnóstica de $100 \%$ dos casos. Uma alternativa para minimizar esta questão seria verificar se outros parâmetros relacionados com neoplasias também estariam alterados como, por exemplo, o IMC, correlacionado como estado nutricional. Entretanto, tal variável não apresentou diferença significativa entre sobreviventes e idosos longevos que foram a óbito no período investigado. Consulta nas estatísticas de mortalidade do Sistema Único de Saúde indicou a morte de 19 indivíduos por algum tipo de neoplasia com idade $\geq 80$ anos no período do estudo. Porém, é preciso considerar que, para o segundo semestre de 1996 e para os anos de 1997, 1998 e primeiro semestre de 1999, outros indivíduos da população chegaram aos 80 anos de idade, e possivelmente estejam incluídos nesta estatística, não representando somente a coorte de idosos longevos investigada. De qualquer modo, a possibilidade de associação entre tais doenças e níveis baixos de CT, LDL-C e ApoA-I não pode ser descartada, existindo a necessidade de estudos complementares.

A associação entre níveis de ApoA-I e mortalidade em idosos também tem sido descrita em estudos prévios. Entre estes, podemos destacar o estudo realizado por Campbell et al. (1993) na população idosa de Mosgiel, zona rural da Nova Zelândia. Foram analisados 515 indivíduos, cuja média de idade foi de 77,5 $\pm 5,4$ anos. Dos 240 indivíduos com 80 anos ou mais, 161 foram classificados como isquêmicos. Os 79 participantes com 80 anos ou mais sem DAC clínica ou alterações isquêmicas ao ECG foram denominados “sobreviventes". Quando comparados aos demais grupos, os "sobreviventes" caracterizaram-se por apresentar níveis significativamente mais elevados de HDL-C e ApoAI, além de menor IMC e níveis mais baixos de triglicerídio (TG). Mesmo quando realizada análise multivariada controlando idade, sexo,
IMC, relação C/Q, atividade física, ingestão de álcool e história de tabagismo, permaneceram diferenças significativas em relação aos níveis de HDL-C $(p=0,05)$ e de ApoA-I $(p=0,02)$. Os autores sugeriram que níveis elevados de ApoA-I e de HDL-C, níveis baixos de TG e IMC próximo do normal poderiam representar importantes fatores determinantes de sobrevida. Desta forma, sugere-se que a associação entre longevidade e ApoA-I elevada ocorra também em outras populações de idosos, como a de Veranópolis, não sendo apenas um efeito de corte do estudo de Campbell et al. (1993).

A literatura sugere que alterações eletrocardiográficas, bem como história prévia de eventos coronarianos ou cerebrovasculares apresentam risco mais elevado para morte vascular, assim como para um novo evento cardíaco ou cerebral em relação aos indivíduos sem estes precedentes (Antiplatelet Trialist's Collaboration, 1988).

Entretanto, no presente estudo, quando analisamos a variável cardiopatia, observamos que a resposta positiva ao Protocolo de Rose não foi significativamente associada à mortalidade e que eram as alterações eletrocardiográficas, BRD e HVE, que tornavam a variável cardiopatia preditora de mortalidade para idosos longevos. Estes achados estão em consonância com os resultados do The Framingham Heart Study (Kannel et al., 1987), do The Belgian Inter-University Reseach on Nutrition and Health Study (BIRNH Study) (Bacquer et al., 1998) e do The Cardiovascular Health Study (Fried et al., 1998), no qual as alterações eletrocardiográficas, e não a história de cardiopatia isquêmica, foram associadas com a mortalidade.

Conforme revisado por Friedmann \& Grindler (1999), a incidência de alterações eletrocardiográficas aumenta com a idade, sendo que o ECG anormal, geralmente, indica cardiopatia clínica ou anatômica. Os distúrbios da condução intraventricular adquiridos em idade avançada são geralmente causados por cardiopatia isquêmica, degeneração senil dos feixes de condução ou evolução tardia da HVE em hipertensos. Embora o BRD possa surgir independentemente de manifestação clínica de doença, fortes evidências sugerem que represente doença anatômica do coração em idosos. As manifestações clássicas de cardiopatia isquêmica tendem a se tornar menos freqüentes entre os idosos (Kuller et al., 1995) e isto poderia explicar a falta da associação entre o Protocolo de Rose e a mortalidade.

Nossos resultados evidenciaram que a presença prévia de doença circulatória, no caso, cardiopatias e AVC, assim como a presença de 
alterações eletrocardiográficas de BRD ou de HVE foram marcadores independentes para mortalidade em indivíduos acima de 80 anos.

A presença de possível viés não pode ser descartada, uma vez que a coorte analisada é constituída de um número pequeno de indivíduos, e que o envelhecimento biológico apresenta uma enorme heterogeneidade que pode dificultar a detecção de preditores reais e reproduzíveis de risco de mortalidade em idosos longevos. Entretanto, como, de modo geral, os resultados obtidos neste trabalho estão de acordo com os de outros estudos populacionais que investigaram indivíduos do mesmo grupo etário, pode-se supor que fatores de risco cardiovascular clássicos atuem de forma diferenciada na população com idade $>80$ anos. Se isto for verdadeiro, considerando-se que existirá um aumento de idosos longevos nas próximas décadas devido ao fenômeno de envelhecimento populacional, e que doenças cardiovasculares são a principal causa de mortalidade neste grupo etário, os dados descritos na literatura e aqui obtidos são de relevância para a clínica cardiológica e geriátrica. Para tanto, seria interessante que outros estudos neste grupo etário fossem produzidos no Brasil, e comparados entre si.

\section{Agradecimentos}

Agradecemos a toda a equipe que participou da fase inicial de coleta de dados em 1996: Dra. Andréia da Costa, Dr. Ariovaldo Pires, Dr. Cristián Mercado, Dr. Rodolfo Schneider, Dr. Maurício Friedrich e Enfermeira Maria Cristina Santana da Silva. Também gostaríamos de agradecer ao prefeito e aos secretários municipais de Saúde e do Trabalho e Ação Social, e, em especial, aos funcionários das referidas secretarias todo o engajamento na pesquisa. Aos pesquisadores do Programa Gênesis de Pesquisa do Instituto de Geriatria e Gerontologia da Pontifícia Universidade Católica do Rio Grande do Sul, que viabilizaram a reestruturação do banco de dados do Projeto Veranópolis, e ao Prof. Glauco Schneider Rolim, pela revisão do artigo.

\section{Referências}

ALENCAR, Y. M. G.; CARVALHO FILHO, E. T.; PASCHOAL, S. M. P.; CURIATI, J. A. E.; PING, W. C. \& LITVOC, J., 2000. Fatores de risco para aterosclerose em uma população idosa ambulatorial na cidade de São Paulo. Arquivos Brasileiros de Cardiologia, 74:181-188.

ANTIPLATELET TRIALIST'S COLLABORATION, 1988. Secondary prevention of vascular disease by prolonged antiplatelet treatment. BMJ, 296:320-331.

BACQUER, D. D.; BACKER, G. D.; KORNITZER M. \& BLACKBURN, H., 1998. Prognostic value of ECG findings for total, cardiovascular disease, and coronary heart disease death in men and women. Heart, 80:570-577.

CAMPBELL, A. J.; BUSBY, W. J. \& ROBERTSON, M. C., 1993. Over 80 years and no evidence of coronary heart disease: Characteristics of a survivor group. Journal of the American Geriatrics Society, 41:13331338.

CASIGLIA, E.; MAZZA, A.; TIKHONOFF, V.; SCARPA, R.; GLUGLIELMI, F. \& PESSINA, A., 2002. Arterial Hypertension and mortality in the elderly. American Journal of Hypertension, 15:958-966. 
CONTOIS, J. H.; McNAMARA, J. R.; LAMMI-KEEFE, C. J.; WILSON, P. W. F.; MASSOV, T. \& SCHAEFER, E. J., 1996a. Reference intervals for plasma apolipoprotein A-I determined with a standardized commercial immunoturbidimetric assay: Results from the Framingham Offspring Study. Clinical Chemistry, 42:507-514.

CONTOIS, J. H.; McNAMARA, J. R.; LAMMI-KEEFE, C. J.; WILSON, P. W. F; MASSOV, T. \& SCHAEFER, E. J., 1996b. Reference intervals for plasma apolipoprotein B determined with a standardized commercial immunoturbidimetric assay: Results from the Framingham Offspring Study. Clinical Chemistry, 42(4):515-523.

DATASUS (Departamento de Informática do SUS), 1996. Informações sobre Mortalidade, 1996. <http: //www.datasus.gov.br>.

DIPIETRO, L.; CASPERSEN, C. J.; OSTFELD, A. M. \& NADEL, E. R., 1993. A survey for assessing physical activity among older adults. Medicine and Science in Sports and Exercise, 25:628-642.

FRIED, L. P.; KRONMAL, R. A.; NEWMAN, A. B.; BILD, D. E.; MITTELMARK, M. B. \& POLAK, J. F., 1998. Risk factors for 5-year mortality in older adults. The cardiovascular health study. JAMA, 279:585592.

FRIEDMANN, A. A. \& GRINDLER, J., 1999. Aplicações clínicas do eletrocardiograma no idoso. Revista da Sociedade de Cardiologia do Estado de São Paulo, 3:286-292.

GRUEN, D. G. \& CONNOR, E. B., 1996. Sex differences in measures of body fat and body fat distribution in the elderly. American Journal of Epidemiology, 143:898-906.

HAYFLICK, L., 1994. How and Why We Age. New York: Ballantine Books.

IBGE (Fundação Instituto Brasileiro de Geografia e Estatística), 2002. Informações sobre a População, 1996. <http://www.ibge.gov.br>.

KANNEL, W. B. \& D'AGOSTINO, R. B., 1997. Blood pressure and cardiovascular morbidity and mortality rates in the elderly. American Heart Journal, 134:758-763.

KANNEL, W. B.; DANNENBERG, A. L. \& LEVY, D., 1987. Population implications of electrocardiographic left ventricular hypertrophy. American Journal of Cardiology, 60:85I-93I.

KASHYAP, M. L., 1989. Cardiovascular disease in the elderly: Current considerations. American Journal of Cardiology, 63:3H-4H.

KRONMAL, R. A.; CAIN, K. C.; YE, Z. \& OMENN, G. S., 1993. Total serum cholesterol levels and mortality risk as a function of age. A report based on the Framingham data. Archives of Internal Medicine, 153:1065-1073.
KULLER, L. H.; SHEMANSKI, L.; PSATY, B. M.; BORHANI, N. O.; GARDIN, J. \& HAAN, M. N., 1995. Subclinical disease as an independent risk factor for cardiovascular disease. Circulation, 92:720-726.

LARSSON, B.; SVÄRDSUDD, K.; WELIN, L.; WILHELMSEN, L.; BJÖRNTORP, P. \& TIBBLIN, G., 1984. Abdominal adipose tissue distribution, obesity, and risk of cardiovascular disease and death: 13 year follow up of participants in the study of men born in 1913. BMJ, 288:1401-1404.

MATTILA, K.; HAAVISTO, M.; RAJALA, S. \& HEIKINHEIMO, R., 1988. Blood pressure and five year survival in the very old. $B M J, 296: 887-889$.

MENCKEN, H. L., 2000. There is a non-linear relationship between mortality and blood pressure. European Heart Journal, 21:1635-1638.

NÚCLEO DE INFORMAÇÃO EM SAÚDE, 1998. Estatísticas de Saúde: Mortalidade de 1997. Porto Alegre: Núcleo de Informação em Saúde, Secretaria de Saúde e do Meio Ambiente do Estado do Rio Grande do Sul.

ROSE, G. A.; BLACKBURN, H.; GILLUM, R. F. \& PRINEAS, R. J., 1982. Cardiovascular Survey Methods. 2nd Ed. Geneva: World Health Organization.

SCHWANKE, C. H. A.; DA CRUZ, I. B. M.; LEAL, N. F; SCHEIBE, R.; MORIGUCHI, Y. \& MORIGUCHI, E. H., 2002. Analysis of the association between apolipoprotein E polymorphism and cardiovascular risk factors in an elderly population with longevity. Arquivos Brasileiros de Cardiologia, 78:571-579.

SOCIEDADE BRASILEIRA DE HIPERTENSÃO/SOCIEDADE BRASILEIRA DE CARDIOLOGIA/SOCIEDADE BRASILEIRA DE NEFROLOGIA, 1998. III Consenso Brasileiro de Hipertensão Arterial. Revista Brasileira de Cardiologia, 1:92-133.

SOIBELMAN Jr., M. E. L., 1990. Problemas relacionados ao consumo de álcool. In: Medicina Ambulatorial (S. G. Duncan, org.), p. 289, Porto Alegre: Atheneu.

TERVAHAUTA, M.; PEKKANEN, J.; KIVINEN, P.; STENGARD, J.; JAUHIAINEN, M. \& EHNHOLM, C., 1993. Prevalence of coronary heart disease and associated risk factors among elderly finnish men in the seven countries study. Atherosclerosis, 104:47-59.

WEVERLING-RIJNSBURGER, A. W. E.; BLAUW, G. J.; LAGAAY, A. M.; KNOOK, D. L.; MEINDERS, A. E. \& WESTENDORP, R. G. J., 1997. Total cholesterol and risk of mortality in the oldest old. Lancet, 350:1119-1123.

Recebido em 18 de abril de 2002

Versão final reapresentada em 13 de janeiro de 2003 Aprovado em 18 de fevereiro de 2003 